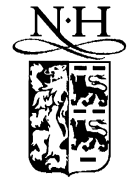

ELSEVIER

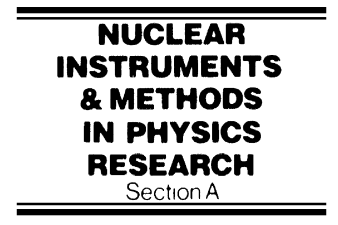

www.elsevier.nl/locate/nima

\title{
A spark-protected high-rate detector
}

\author{
P. Fonte ${ }^{\mathrm{a}, \mathrm{b}, *}$, N. Carolino ${ }^{\mathrm{a}}$, L. Costa ${ }^{\mathrm{c}}$, Rui Ferreira-Marques ${ }^{\mathrm{a}, \mathrm{d}}$, S. Mendiratta ${ }^{\mathrm{c}}$, \\ V. Peskov ${ }^{\mathrm{e}, 1}$, A. Policarpo ${ }^{\mathrm{a}, \mathrm{d}}$ \\ ${ }^{a}$ LIP, Dep. de Fisica, Univ. de Coimbra, 3000 Coimbra, Portugal \\ ' ISEC, Quinta da Nora, 3000 Coimbra, Portugal \\ ${ }^{\mathrm{c}}$ Departamento de Fisica, Univ. de Aveiro, 3800 Aveiro, Portugal \\ ${ }^{\mathrm{d} D e p a r t a m e n t o ~ d e ~ F i s i c a, ~ U n i v . ~ d e ~ C o i m b r a, ~} 3000$ Coimbra, Portugal \\ ${ }^{\mathrm{e}}$ NASA Marshall Space Flight Center, Huntsville, AL 35812, USA
}

Received 15 January 1999

\begin{abstract}
We developed a very low resistivity RPC-type detector, the anode of which was a plate made from materials with resistivity up to $5 \times 10^{7} \Omega \mathrm{cm}$, the cathode being a metallic mesh preceded by a drift region.

In such a detector it was actually possible to combine the versatility and high counting-rate capability of metallic PPACs with the extreme robustness and "protectiveness" of Resistive Plate Chambers.

Occasional discharges triggered by large deposits of primary ionisation or by extreme counting rates are quenched by the resistive anode and are constrained to the streamer phase of the sparking process. The study shows that this discharge affects the detector only locally and that the charge released is limited to a few tens of $\mathrm{nC}$.

Proportional counting rates up to $10^{5} \mathrm{~Hz} / \mathrm{mm}^{2}$ were achieved at gains above $10^{4}$. The energy resolution at $6 \mathrm{keV}$ was $20 \%$ FWHM. The observed gain-rate trade-off is well described by an analytic model and further improvements may be expected by lowering the resistivity of the anode material.

The properties of several custom-made, controllable resistivity, anode materials are described and prospects of improvement in the performance of the detector are discussed. (C) 1999 Elsevier Science B.V. All rights reserved.
\end{abstract}

\section{Introduction}

Parallel geometry chambers are used in three main configurations: Parallel Plate Chambers

\footnotetext{
* Corresponding author. Present address: CERN/EP, CH-1211, Geneve 23, Switzerland.

E-mail address: paulo.fonte@cern.ch, fonte@lipc.fis.uc.pt (P. Fonte)

${ }^{1}$ Present address: Phys. Dept. in Frescati, Royal Institute of Technology, Frescativagen 24, Stockholm S-10405, Sweden.
}

(PPCs), with two solid metallic electrodes, Resistive Plate Chambers (RPCs), with two resistive electrodes and Parallel Mesh Chambers ${ }^{2}$ (PMCs), with multiple wire-mesh electrodes [1].

Common features of these detectors when operated in proportional mode are the good timing

\footnotetext{
${ }^{2}$ Also known historically as Parallel Plate Avalanche Chambers (PPACs), although in these detectors there are no plate electrodes.
} 
characteristics $[2,13,14]$, good position resolution $[3,23]$, ease of production in large areas and large gain. The maximum gain is limited to the point where individual avalanches reach a charge around $10^{8}$ electrons, triggering the formation of streamers $[4,15-17]$ that subsequently evolve to higher current discharges [5]. Recently a new rate-induced breakdown mode was identified in PMCs [6].

In chambers with metallic electrodes (PPCs, PMCs) the discharge progresses until fully formed sparks appear. Although the sparks do not seem to affect the detector in a permanent way [7], the phenomenon causes unacceptable dead times and may compromise the integrity of the readout electronics.

When the electrodes are highly resistive (RPCs) the discharge is quenched at an earlier stage, affecting the detector only locally and being harmless to the readout electronics. It is even possible to operate the RPCs in a saturated gain "streamer mode", featuring very large and fast signals, up to a few hundred pC $[8,18,19]$.

The advantage of RPCs in terms of sparkless operation is offset by a severe counting rate limitation of a few tens of $\mathrm{Hz} / \mathrm{mm}^{2}$, while PPCs and PMCs can reach counting rates at least up to $10^{5} \mathrm{~Hz} / \mathrm{mm}^{2}[6,9,20]$ without any decrease of the pulse-height, suggesting their use as high-rate detectors.

It is attractive to attempt to combine the "protectiveness" of RPCs with the large counting rate capability of the metallic chambers. Earlier attempts $[10,21]$ were focused on an asymmetric RPC with wire-mesh electrodes except for a resistive anode made of Pestov glass. However due to a lack of anode materials with lower resistivity, the counting rate of the device did not exceed a few hundreds of $\mathrm{Hz} / \mathrm{mm}^{2}$.

In the present work we introduce a new custommade medium resistivity material whose resistivity can be controlled from $2 \times 10^{7}$ to $3 \times 10^{12} \Omega \mathrm{cm}$ allowing for a large flexibility in the chamber counting characteristics, while keeping essentially the same hybrid configuration described above.

The resulting detector actually combines the high counting-rate capability of PMCs $\left(10^{5} \mathrm{~Hz} / \mathrm{mm}^{2}\right)$ with the extreme robustness and "protectiveness" of RPCs.

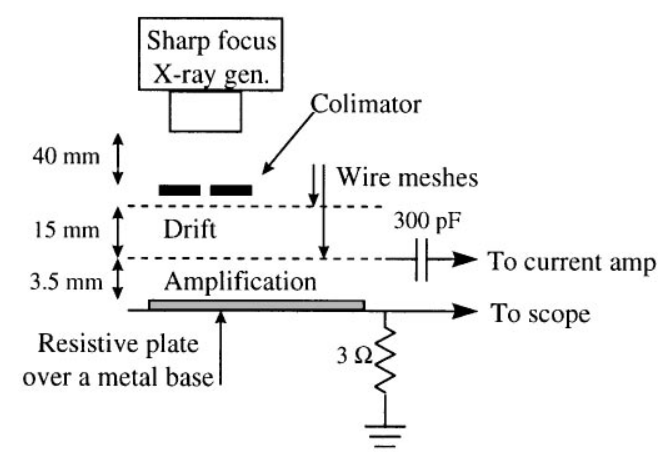

Fig. 1. Schematic representation of the experimental setup.

\section{Experimental setup}

A schematic drawing of the experimental setup can be seen in Fig. 1.

The detector was constituted by a drift region followed by an amplifying gap. The drift region was $15 \mathrm{~mm}$ long and delimited by two woven-wire meshes. The amplifying gap, $3.5 \mathrm{~mm}$ long, was delimited by the lower drift mesh (cathode) and by a resistive plate (anode). The meshes were made of stainless steel wires of $50 \mu \mathrm{m}$ diameter placed at a pitch of $0.5 \mathrm{~mm}$. The detector had an active diameter of $4 \mathrm{~cm}$.

The primary charges were created by a collimated X-ray beam produced by a sharp-focus $(0.1 \mathrm{~mm}) \mathrm{X}$-ray tube having a $\mathrm{Fe}$ anode. The tube produces a bremstrahlung photon energy distribution peaked at $5.5 \mathrm{keV}$ with a maximum energy of $10 \mathrm{keV}$. The beam was filtered by a nickel foil to absorb the lowest energy photons and delimited by a flat ring collimator placed $4 \mathrm{~cm}$ from the tube anode, with selectable openings of 2 and $5 \mathrm{~mm}$ diameter. The collimator was placed over the Mylar entrance window, $1 \mathrm{~mm}$ from the upper drift mesh. It is estimated that the $5.5 \mathrm{keV}$ photons will generate 200 primary electrons, and this was the value used for all calculations.

The resistive plate was deposited over a flat metal plate that provided mechanical support and a uniform electrical connection to the plate. The metal plate was grounded through a $3 \Omega$ resistor and the current signal from discharges could be 


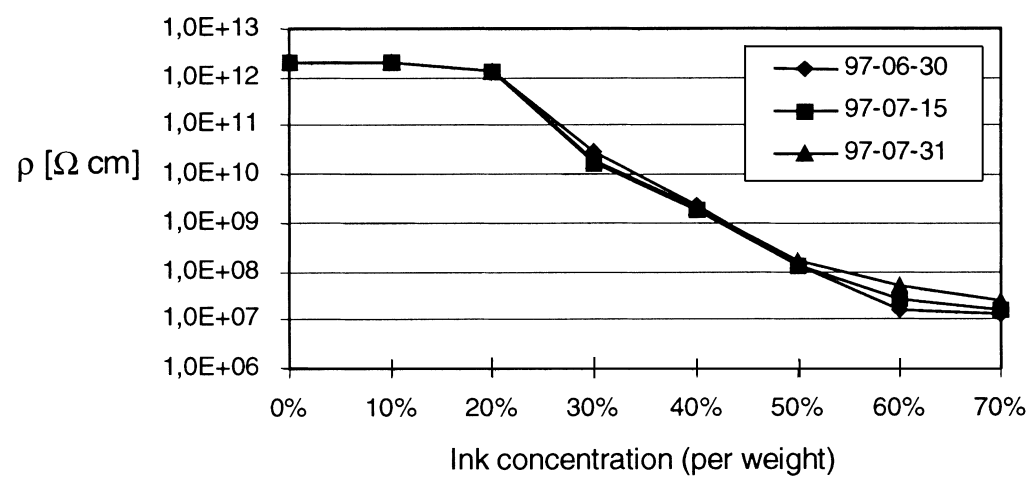

Fig. 2. The anodic material resistivity can be controlled by the amount of ink and varied from $2 \times 10^{7}$ to $3 \times 10^{12} \Omega \mathrm{cm}$. Successive measurements taken at two week intervals suggest a good stability of the bulk resistivity.

recorded directly on a scope connected in parallel with the grounding resistor.

The cathode signal was readout through a capacitor by a fast current amplifier with $30 \mathrm{~ns}$ rise time. Since the rise time of the amplifier was actually longer than the signal width, the pulse height at the amplifier output was related to the avalanche charge and not to the gap current. It was found that the observed pulse shape at the amplifier output was well reproduced by injecting in parallel with the amplifying gap a square current pulse of $10 \mathrm{~ns}$ width. When the amplitude was $160 \mu \mathrm{A}$ this pulse corresponded to the injection of $10^{7}$ electrons or $1.6 \mathrm{pC}$, thus providing a charge calibration of the readout system.

In order to measure the overall counting rate the output of the fast current amplifier was sensed by a discriminator whose trigger rate was measured by a time-windowed scaler. The peak noise level at the amplifier output was around $25 \mathrm{fC}$ and the discriminating threshold for all measurements was placed at $80 \mathrm{fC}$. This relatively high level was forced by a noisy X-ray tube power supply.

The gas mixture was $\mathrm{Ar}+20 \% \mathrm{C}_{2} \mathrm{H}_{6}+$ methanol. The mixture was $50 \%$ saturated with the methanol vapours, that were required to prevent the resistive plate to become dry, with consequent increase of the plate surface resistivity.

\section{Resistive material}

The anodic resistive plate was made from a mixture of epoxy ${ }^{3}$ and ink ${ }^{4}$ that yields a black rubberlike material.

The resistivity can be varied from $2 \times 10^{7}$ to $3 \times 10^{12} \Omega \mathrm{cm}$ by varying the ink concentration (Fig. 2). Successive measurements taken at two weeks intervals suggest a good stability of the bulk resistivity. Additionally the material shows an ohmic behaviour up to an applied voltage of $1 \mathrm{kV}$.

Further measurements have been performed in a systematic way trying to identify materials of potential interest for this type of application. In particular, Fig. 3 shows results for melamine and for the above material, together with ABS plastic doped with $\mathrm{FeCl}_{3}$. The latter seems to be a promising candidate, having much better mechanical characteristics than the epoxy-ink mixture.

\section{Results and discussion}

\subsection{Counting characteristics}

The detector shows a reasonable energy resolution of $20 \%$ FWHM for $5.9 \mathrm{keV}$ X-rays, while the

\footnotetext{
${ }^{3}$ Araldite.

${ }^{4}$ MOLIN, ball-point pen black ink.
} 


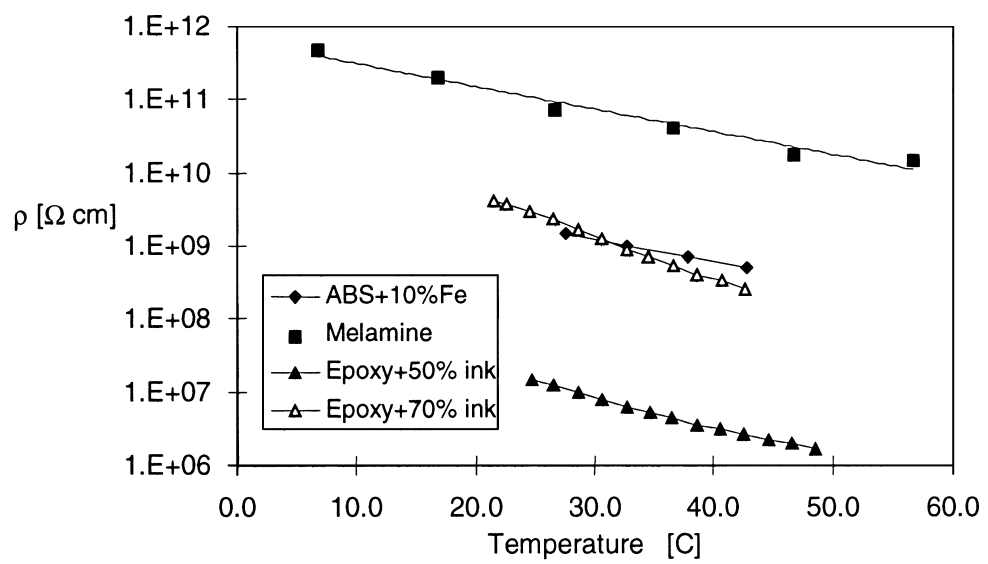

Fig. 3. Resistivity measurements performed for various materials in a temperature range of practical interest.

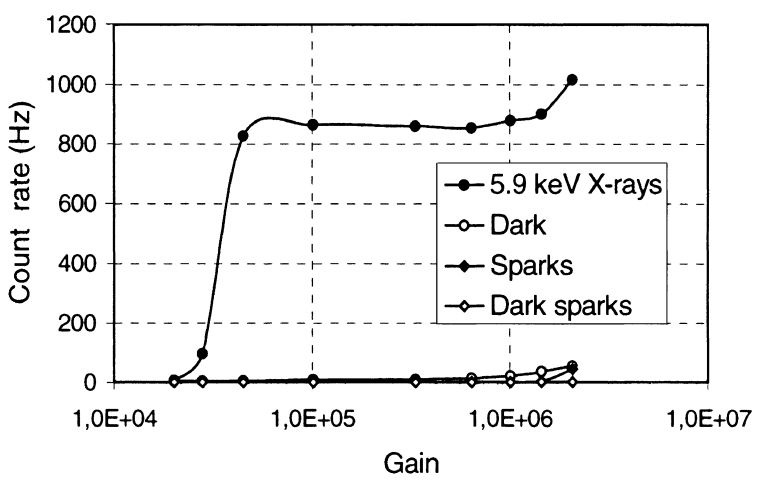

Fig. 4. A large counting plateau of more than one order of magnitude in gain was observed when the detector was illuminated by $5.9 \mathrm{keV} \mathrm{X}$-rays. The corresponding dark current is negligible and discharges appear only at gains above $10^{6}$.

best results for PMCs are typically around $14 \%$ FWHM [11]. In principle there is no reason why the present detector cannot achieve similar resolution values, so we attribute the observed reduced resolution to the deficient parallelism of the gap and to edge effects arising from the relatively small diameter of the active area.

A large counting plateau of more than one order of magnitude in gain was observed when the detector was illuminated by $5.9 \mathrm{keV}$ X-rays (Fig. 4). The corresponding dark current is negligible and sparks appear only at gains in excess of $10^{6}$. The dark current was found to be extremely sensitive to the presence of dust particles, requiring the detector to be assembled in a dust-free environment.
The gain-rate characteristics of the detector were studied for several combinations of applied voltages, plate materials and beam diameters, the detector being illuminated with $\mathrm{X}$ photons generated by the tube described in Section 2. The results are shown in Fig. 5.

For all plate materials, with resistivity ranging from $4 \times 10^{7}$ to $4 \times 10^{11} \mathrm{~cm}$, a reduction in gain was observed above a certain rate threshold. The threshold rate is different for each plate, but seems to be relatively unaffected by the operating voltage or by the beam diameter.

For the lower plate resistivity studied, counting rates of $10^{5} \mathrm{~Hz} / \mathrm{mm}^{2}$ were achieved at gains between $10^{4}$ and $10^{5}$. This value is actually slightly above the intrinsic rate-gain limitations that were found in similarly built metallic PMCs [6], indicating that the optimum anode resistivity has been reached. The fact that in RPCs one can reach higher gains than in metallic chambers is well established in the literature, this fact being probably due to the "protectiveness" provided by the resistive electrodes.

The simplest assumption one can make about the origin of the rate-gain dependence is that it is caused by a reduction of the effective gap electric field owing to an ohmic voltage drop across the anode plate when crossed by the avalanche currents. An analytic model based on this effect was developed, and the predicted rate-gain dependence was plotted in Fig. 5 (thin lines). It can be seen that the model describes reasonably well the observed behaviour. 


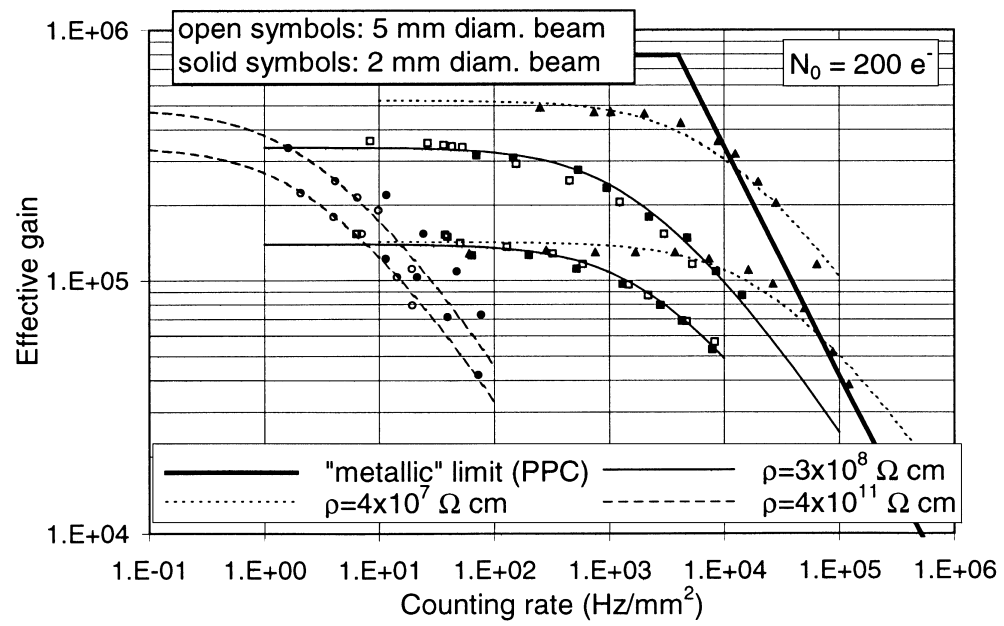

Fig. 5. Gain-rate characteristics of the detector for several values of the anode plate resistivity and beam diameters of 2 and 5 mm. For the lower resistivity studied counting rates of $10^{5} \mathrm{~Hz} / \mathrm{mm}^{2}$ were achieved at gains between $10^{4}$ and $10^{5}$. The thin lines were adjusted according to an analytic ohmic model and the thick solid line marks the intrinsic counting rate limitations of a similarly built all-metallic PMC [6].

Table 1

Charge released by the discharges $\left(Q_{\text {discharge }}\right)$ for different values of the plate resistivity and plate thickness $(L)$

\begin{tabular}{lll}
\hline$\rho(\Omega \mathrm{cm})$ & $L(\mathrm{~mm})$ & $Q_{\text {discharge }}(\mathrm{nC})$ \\
\hline $4 \times 10^{11}$ & 0.25 & 33 \\
$3 \times 10^{8}$ & 1 & 28 \\
$4 \times 10^{7}$ & 1.5 & 21 \\
\hline
\end{tabular}

\subsection{Streamer charge}

Discharges were triggered by streamers when the gain exceeded $10^{6}$ (see Fig. 4), that is, when the avalanche charge exceeded about $2 \times 10^{8}$ electrons. Eventually some of the highest rate points shown in Fig. 5 correspond to the limit of rate-induced breakdown [6], but this was not directly verified experimentally.

In Table 1 we show the measured values of the charge released by the discharges $\left(Q_{\text {discharge }}\right)$ for each resistive plate used. The charge released was limited to a few tens of $\mathrm{nC}$.

For the lower resistivity material $(\rho=$ $\left.4 \times 10^{7} \Omega \mathrm{cm}\right)$ sometimes the discharge would be unquenched, fed by the conduction current across the plate. An increase in the amount of quencher $\left(\mathrm{C}_{2} \mathrm{H}_{6}\right)$ in the gas mixture strongly reduced the frequency of this phenomenon, with out completely avoiding it. However it seems probable that the use of more efficient quenchers like iso-butane or DME would further alleviate the problem.

\section{Conclusions}

We built and tested an asymmetric Resistive Plate Chamber able to achieve proportional counting rates up to $10^{5} \mathrm{~Hz} / \mathrm{mm}^{2}$ at gains between $10^{4}$ and $10^{5}$. The energy resolution for $5.9 \mathrm{keV}$ X-rays was $20 \%$ FWHM.

The chamber was constituted by an amplifying gap delimited by a resistive plate anode and a wire mesh cathode, preceded by a drift region. A new custom-made medium resistivity material was used for the anode plate, whose resistivity could be controlled from $2 \times 10^{7}$ to $3 \times 10^{12} \Omega \mathrm{cm}$.

Eventual discharges were quenched by the current limitation imposed by the resistive anode. The discharge affected the detector only locally and the 
charge released was limited to a few tens of $\mathrm{nC}$, independently of the plate DC resistivity.

The observed gain-rate trade-off is well described by an analytic ohmic model.

Being the characteristic relaxation time of the anodic plate $(\rho \varepsilon)$ of the order of a few $\mu$ s, the electric transparency of the plate is preserved for the fast $(<100 \mathrm{~ns})$ electron signal. This will, in principle, allow a bidimensional strip readout to be placed below the anodic plate, with typical position resolutions of 100-200 $\mu \mathrm{m}$ [3].

Finally, the spark protection concept presented here may be also useful when applied to higherrate detectors like MICROMEGAS or other thin-gap parallel geometry chambers [12,22]. In such a case, lower resistivity materials will be required and further research is therefore needed.

\section{Acknowledgements}

The authors gratefully acknowledge Crispin Williams and the LAA Project for lending some of the necessary hardware. This work was done in the framework of the projects JNICT-CERN/ $\mathrm{P} / \mathrm{FAE} / 1098 / 96$ and JNICT-CERN/P/FAE/ 1143/97.

\section{References}

[1] G. Charpak, F. Sauli, Phys. Lett. 78 B (1978) 523.

[2] E. Ceron Zebalos, Sci. Acta XI-1 (1996) 317.

[3] A. Peisert et al., Nucl. Instr. and Meth. A 247 (1986) 435.

[4] H. Raether, Electron Avalanches and Breakdown in Gases, Butterworths, London, 1964.

[5] S.C. Haydon, in: J.A. Rees (Ed.), Electrical Breakdown of Gases, Macmillan, London, 1973.

[6] Y. Ivaniouchenkov et al., The high rate behaviour of Parallel Mesh Chambers, Presented at the IEEE Nuclear Science Symp., 9-15 November, 1997, Albuquerque, NM, USA, IEEE Trans. Nucl. Sci. 45 (1998) 244.

[7] A. Arefiev et al., Sci. Acta XI-1 (1996) 359.

[8] I. Duerdoth et al., Nucl. Instr. and Meth. A 348 (1994) 303.

[9] A. Peisert, Nucl. Instr. and Meth. 217 (1983) 229.

[10] V. Peskov et al., FERMILAB TM-1838, 1993.

[11] B.D. Ramse et al., Nucl. Instr. and Meth. A 248 (1986) 550.

[12] Y. Giomataris, Nucl. Instr. and Meth A 419 (1998) 239.

[13] A. Arefiev et al., Sci. Acta XI-1 (1996) 359.

[14] M. Angelone et al., Nucl. Instr. and Meth. A 355 (1995) 359.

[15] J.M. Meek, in: J.A. Rees (Ed.), Electrical Breakdown of Gases, Macmillan, London, 1973.

[16] P. Fonte et al., Nucl. Instr. Meth. A 310 (1991) 140.

[17] P. Fonte, IEEE Trans. Nucl. Sci. 43 (1996) 2135.

[18] R. Cardarelli et al., Sci. Acta XI-1 (1996) 11.

[19] P. Fonte, Sci. Acta XI-1 (1996) 25.

[20] J. Hendrix et al., Nucl. Instr. and Meth. A 252 (1986) 246.

[21] D.F. Anderson et al., Nucl. Instr. and Meth. A 348 (1994) 324.

[22] P. Fonte et al., Preprint LIP/97-05, December 1997.

[23] E. Ceron Zebalos, Nucl. Instr. and Meth. A 392 (1997) 150. 